\title{
Stochastic Minimax Vibration Control for Uncertain Nonlinear Quasi-Hamiltonian Systems with Noisy Observations
}

\author{
Zu-guang Ying \\ Department of Mechanics, School of Aeronautics and Astronautics, Zhejiang University, Hangzhou 310027, China.
}

\begin{abstract}
Rong-chun Hu
Department of Engineering Mechanics, Northwestern Polytechnical University, Xi'an 710129, China.
\end{abstract}

\begin{abstract}
Rong-hua Huan
Department of Mechanics, School of Aeronautics and Astronautics, Zhejiang University, Hangzhou 310027, China.
\end{abstract}

\begin{abstract}
(Received 21 October 2017; accepted 7 June 2018)
A stochastic minimax control strategy for uncertain nonlinear quasi-Hamiltonian systems with noisy observations under random excitations is proposed based on the extended Kalman filter and minimax stochastic dynamical programming principle. A structure system with smart sensors and actuators is modeled as a controlled, excited and dissipative Hamiltonian system with noisy observations. The differential equations for the uncertain nonlinear quasi-Hamiltonian system with control and observation under random excitation are given first. The estimated nonlinear stochastic control system with uncertain parameters is obtained from the uncertain quasi-Hamiltonian system with noisy observation. In this case, the optimally estimated state is determined by the observation based on the extended Kalman filter. The dual dynamical programming equation for the estimated uncertain system is then obtained based on the minimax stochastic dynamical programming principle. The worst-case disturbances are determined for bounded uncertain parameters and the optimal control law is determined for the worst case by the programming equation. The proposed minimax control strategy is applied to two uncertain nonlinear stochastic systems with controls and noisy observations. The control effectiveness for the stochastic vibration response reductions of the systems is illustrated with numerical results. The proposed minimax control strategy is applicable to general uncertain nonlinear multi-degree-of-freedom structure systems with noisy observations under random excitations.
\end{abstract}

\section{INTRODUCTION}

The vibration control for engineering structures subjected to strong random excitations is a significant research subject. ${ }^{1-3}$ Smart materials such as piezoelectric and magnetorheological materials have been applied to the structural vibration mitigation. ${ }^{4-20}$ A structure system with smart sensors, actuators and controllers is called a smart structure. A smart structure system can sense structural response to external excitations and produce action to control structural responses. ${ }^{21-23}$ The control performance of the smart structure system depends strongly on its used control strategy. The system control includes the state estimation based on sensing data and the response control based on estimated states, which are coupled with each other. ${ }^{24-28}$ However, the sensing data, or observations and excitations, inevitably have random noise components. Only linear control strategies for smart structure systems with noisy observations have been proposed presently.

The smart structure, including multi-degree-of-freedom systems, beams and plates in modal vibration, can be modeled as a controlled, excited and dissipative Hamiltonian system or quasi-Hamiltonian system with observation. ${ }^{29}$ The quasiHamiltonian system is nonlinear for strong random excitations. The stochastic optimal control for linear and nonlinear systems has been studied and many control strategies have been pre- sented. ${ }^{29-46}$ In general, the separation theorem is used to convert the original system with noisy observations into another system with complete observations and then the optimal control law is determined based on the stochastic dynamical programming principle. However, for the nonlinear stochastic system with noisy observations, the separation theorem yields an estimated system with infinite-dimensional probability density, such that the optimal control cannot be determined. ${ }^{27}$ Only several studies presented the stochastic optimal control for a nonlinear system with noisy observation under very specified condition. A non-trivial solution to the estimation is still difficult to obtain. ${ }^{44}$ Thus, an approximate optimal estimation is an alternative for practice. The extended Kalman filter, as an optimum feasible approximate filter, has been applied to the nonlinear stochastic control system with noisy observation recently. ${ }^{24,28,45,46}$

The analysis system of an actual engineering structure has usually uncertain parameters and external disturbances, which can make the designed control performance degenerate. ${ }^{47,48}$ The control problem on uncertain systems can be resolved according to a robust control strategy. ${ }^{49}$ The uncertain optimal control is generally treated as a minimax control problem, in which the minimization is taken over all control strategies and the maximization is taken over possible uncertain- 
ties. $^{50}$ The robust control for deterministic linear and nonlinear systems with uncertain disturbances has been studied extensively. ${ }^{49-51}$ The stochastic robustness of linear control systems was analysed. ${ }^{52}$ The minimax control for linear stochastic uncertain systems was proposed by optimizing the worst-case performance under certain uncertainty constraints and separating estimator and controller. ${ }^{53-56}$ The minimax control for nonlinear stochastic uncertain systems was proposed based on the stochastic differential game theory for bounded uncertainties. ${ }^{57,58}$ However, the system state estimation and observation have not been considered in the minimax nonlinear stochastic control strategy. Thus, the minimax vibration control for uncertain smart structure systems, or uncertain nonlinear quasiHamiltonian systems, with noisy observations under random excitations needs to be studied further, for example, based on the extended Kalman filter and stochastic minimax control strategy which includes the stochastic differential game theory and stochastic dynamical programming principle.

This paper studied the stochastic minimax control for the vibration response reduction of uncertain nonlinear quasiHamiltonian systems with noisy observations. A new stochastic minimax control strategy for the uncertain quasiHamiltonian systems was proposed based on the extended Kalman filter and minimax stochastic dynamical programming principle. First, the structure systems with smart sensors and actuators were modeled as controlled, excited and dissipative Hamiltonian systems with noisy observations. The differential equations for the uncertain nonlinear quasi-Hamiltonian systems with controls and observations under random excitation were given. Second, the extended Kalman filter was applied to convert the quasi-Hamiltonian systems with noisy observations into the completely observed nonlinear stochastic control systems with uncertain parameters for the estimated state. Third, the minimax stochastic dynamical programming principle was applied to derive the dual dynamical programming equation. The worst-case disturbances were determined for bounded uncertain parameters, and then the optimal control law was determined for the worst case and estimated state by the programming equation. Finally, the proposed minimax control strategy was applied to two nonlinear stochastic systems with controls and noisy observations. Numerical results were given to illustrate the control effectiveness.

\section{VIBRATION EQUATIONS OF UNCERTAIN QUASI-HAMILTONIAN SYSTEM WITH NOISY OBSERVATION}

Smart materials such as piezoelectric material have been applied recently to the vibration control of engineering structures under random excitations. A structure with smart devices can sense structural response to external excitations and produce action to control structural response. The feedback control is based on the state observation, or sensing response. The state estimation needs to be considered first for the response control. The basic equations for main structure, actuator structures and sensor structures have been given by. ${ }^{45,46,59,60}$ These equations can be transformed into dynamic equations in the form of second-order differentials, with respect to time, by using structural dominant modes. The equations are commonly expressed as Lagrangian equations. However, for optimal estimation and control, the Lagrangian equations need to be con- verted into other equations in the form of first-order differentials. Generalized Hamiltonian equations are an optimum option. The Hamiltonian generally represents system energy and the stochastic averaging method can be applied to evaluate system response. Thus, the main structure with actuators was expressed as quasi-Hamiltonian equations with control, excitation and dissipative forces. The sensor structure was expressed as an observation equation, in which the inertia force was small and neglected. Actual engineering structures have inevitably uncertain parameters and external disturbances, which can make the control performance degenerate and need to be considered. The differential equation of the uncertain quasi-Hamiltonian system with $n$ degree-of-freedoms was given by: ${ }^{45}$

$$
\dot{\mathbf{X}}=\mathbf{J} \frac{\partial H_{a}}{\partial \mathbf{X}}+\mathbf{C X}+\boldsymbol{\Delta}(\mathbf{X}, t)+\mathbf{B} \Phi_{a}+\mathbf{F W}(t)
$$

where $H_{a}$ was the Hamiltonian. $\mathbf{X}$ was the $2 n$-dimensional state vector. J was the unit symplectic matrix. C was the damping coefficient matrix. $\boldsymbol{\Delta}$ was the uncertain component vector including uncertain stiffness, damping and excitation. B was the control coefficient matrix. $\boldsymbol{\Phi}_{a}$ was the $n_{a^{-}}$ dimensional control vector. $\mathbf{F}$ was the excitation coefficient matrix and $\mathbf{W}$ was the $m$-dimensional unit intensity excitation vector, which is considered as Gaussian white noise. The equation of the observation system was $^{45}$ :

$$
\boldsymbol{\Phi}_{s}=\mathbf{D X}+\mathbf{E W}_{s}(t)
$$

where $\boldsymbol{\Phi}_{s}$ was the $n_{s}$-dimensional observation vector. $\mathbf{W}_{s}$ was the $m_{s}$-dimensional Gaussian white noise vector with unit intensity. $\mathbf{D}$ and $\mathbf{E}$ were the coefficient matrices. The observation noise was independent of the white noise excitation.

Equations (1) and (2) describe an uncertain nonlinear quasiHamiltonian system with control and noisy observation under random excitation. The system state was estimated by using Eq. (2). The estimated state was used to determine the feedback control for system (1). Both the optimal estimation and control needed to be considered for the nonlinear stochastic system. For the system uncertainty, the minimax strategy was used to determine the optimal control for the worst case.

\section{STOCHASTIC OPTIMAL ESTIMATION AND CONTROL BASED ON MINIMAX STRATEGY}

The optimal control of the nonlinear stochastic system (1) with observation (2) included the optimal state estimation and the optimal response control based on the estimated state. The optimal estimation was to find an accurate state by minimizing the estimated error. The error index for observation (2) was expressed as:

$$
J_{F}(\hat{\mathbf{X}})=E\left[l_{F}(\mathbf{X}-\hat{\mathbf{X}}) \mid \mathbf{\Phi}_{s}\right]
$$

where $\hat{\mathbf{X}}$ was the estimated state. $E[\cdot]$ was the expectation operator and $l_{F}(\cdot) \geq 0$ was a continuous differentiable function, such as a quadratic function. For the nonlinear stochastic system (1) and observation (2) with index (3), the optimally estimated state probability density with infinite dimensions was 
generally difficult to obtain exactly. Following this, the extended Kalman filter, ${ }^{24,28}$ as an optimum feasible approximate filter, was applied to yield the approximate estimation. Based on the extended Kalman filter, the nonlinear stochastic differential equation for the estimated state was obtained from system (1) with observation (2) and index (3) as $^{24}$ :

$$
\begin{aligned}
\dot{\hat{\mathbf{X}}}=\mathbf{J} \frac{\partial H_{a}(\hat{\mathbf{X}})}{\partial \hat{\mathbf{X}}}+ & \mathbf{C} \hat{\mathbf{X}}+\boldsymbol{\Delta}_{s}(\hat{\mathbf{X}})+\mathbf{B} \Phi_{a} \\
& +\mathbf{R D}^{T} \mathbf{R}_{s}^{-1} \mathbf{W}_{I}(t)+\boldsymbol{\Delta}_{e} \mathbf{W}_{u}(t)
\end{aligned}
$$

where $\mathbf{W}_{I}$ was the Gaussian white noise vector with covariance matrix $\mathbf{R}_{s}=\mathbf{E} \mathbf{E}^{T}$, and $\mathbf{R}$ was the covariance matrix of the estimated error $\tilde{\mathbf{X}}=\mathbf{X}-\hat{\mathbf{X}}$, which was determined by:

$$
\begin{aligned}
\dot{\mathbf{R}}=\left\{\mathbf{J} \frac{\partial^{2} H_{a}(\hat{\mathbf{X}})}{\partial \hat{\mathbf{X}}^{2}}+\right. & \mathbf{C}\} \mathbf{R}+\mathbf{R}\left\{\mathbf{J} \frac{\partial^{2} H_{a}(\hat{\mathbf{X}})}{\partial \hat{\mathbf{X}}^{2}}+\mathbf{C}\right\}^{T} \\
& +\mathbf{F} \mathbf{R}_{W} \mathbf{F}^{T}-\mathbf{R D}^{T} \mathbf{R}_{s}^{-1} \mathbf{D R}
\end{aligned}
$$

where $\mathbf{R}_{W}=$ I was the identity matrix. The uncertain component vector $\boldsymbol{\Delta}_{s}$ was the part of $\boldsymbol{\Delta}$ that was independent of excitation disturbances. The uncertain component vector $\boldsymbol{\Delta}_{e} \mathbf{W}_{u}$ contained the other $\boldsymbol{\Delta}$ part and estimated error effect. $\mathbf{W}_{u}$ was the Gaussian white noise vector with unit intensity. Therefore, the optimal control of the nonlinear stochastic system (1) with observation (2) had been converted into that of the estimated system (4) with complete observation.

The optimal control was used to find a control law by minimizing certain performance indexes. The performance index for system (4) was expressed as:

$$
J_{C}\left(\boldsymbol{\Phi}_{a}\right)=E\left[\int_{0}^{t_{f}} L_{C}\left(\hat{\mathbf{X}}(t), \boldsymbol{\Phi}_{a}(t)\right) d t+\Psi\left(\hat{\mathbf{X}}\left(t_{f}\right)\right)\right]
$$

where $L_{C}(\cdot) \geq 0$ was a continuous differentiable function. $\Psi\left(t_{f}\right)$ was the terminal cost and $t_{f}$ was the terminal time. Equations (4) and (6) describe the optimal control problem of the nonlinear stochastic system with the estimated state. However, system (4) has uncertain parameters and excitations. Thus, the minimax control strategy ${ }^{50,57}$ was applied to search for an optimal worst-case control, such that the control was robust. The minimax control was described as a stochastic differential game problem with the objective:

$$
\min _{\Phi_{a} \max _{s}, \Delta_{e}} J_{C}\left(\boldsymbol{\Phi}_{a}\right)
$$

According to the minimax stochastic dynamical programming principle, the dynamical programming equation for system (4) with index (6) and strategy (7) was obtained as ${ }^{57}$ :

$$
\begin{gathered}
\frac{\partial V}{\partial t}+\min _{\Phi_{a}} \max _{\Delta_{s}, \Delta_{e}}\left\{\frac{1}{2} \operatorname{tr}\left[\left(\mathbf{R D}^{T} \mathbf{R}_{s}^{-1} \mathbf{D R}+\boldsymbol{\Delta}_{e} \boldsymbol{\Delta}_{e}^{T}\right) \frac{\partial^{2} V}{\partial \hat{\mathbf{X}}^{2}}\right]\right. \\
+\left[\mathbf{J} \frac{\partial H_{a}}{\partial \hat{\mathbf{X}}}+\mathbf{C} \hat{\mathbf{X}}+\Delta_{s}(\hat{\mathbf{X}})+\mathbf{B} \boldsymbol{\Phi}_{a}\right]^{T} \frac{\partial V}{\partial \hat{\mathbf{X}}} \\
\left.+L_{C}\left(\hat{\mathbf{X}}, \boldsymbol{\Phi}_{a}\right)\right\}=0
\end{gathered}
$$

where $V$ was the value function and $\operatorname{tr}(\cdot)$ was the trace operator. In this case, the uncertain parameters in $\boldsymbol{\Delta}_{s}$ and $\boldsymbol{\Delta}_{e}$ were considered as bounded. Following this, the maximization of the left side of Eq. (8) determined the worst-case disturbances:

$$
\begin{aligned}
& \boldsymbol{\Delta}_{s}^{*}=\left.\boldsymbol{\Delta}_{s}\right|_{\max \left\{\boldsymbol{\Delta}_{s}^{T} \partial V / \partial \hat{\mathbf{X}}\right\}} \\
& \boldsymbol{\Delta}_{e}^{*}=\left.\boldsymbol{\Delta}_{e}\right|_{\max \left\{\operatorname{tr}\left[\boldsymbol{\Delta}_{e} \boldsymbol{\Delta}_{e}^{T} \partial^{2} V / \partial \hat{\mathbf{X}}^{2}\right]\right\}} .
\end{aligned}
$$

By using Eq. (9), the dynamical programming equation (8) for the worst-case disturbances became:

$$
\begin{array}{r}
\frac{\partial V}{\partial t}+\min _{\Phi_{a}}\left\{\frac{1}{2} \operatorname{tr}\left[\left(\mathbf{R D}^{T} \mathbf{R}_{s}^{-1} \mathbf{D R}+\boldsymbol{\Delta}_{e}^{*} \boldsymbol{\Delta}_{e}^{* T}\right) \frac{\partial^{2} V}{\partial \hat{\mathbf{X}}^{2}}\right]\right. \\
+\left[\mathbf{J} \frac{\partial H_{a}}{\partial \hat{\mathbf{X}}}+\mathbf{C} \hat{\mathbf{X}}+\Delta_{s}^{*}(\hat{\mathbf{X}})+\mathbf{B} \Phi_{a}\right]^{T} \frac{\partial V}{\partial \hat{\mathbf{X}}} \\
\left.+L_{C}\left(\hat{\mathbf{X}}, \boldsymbol{\Phi}_{a}\right)\right\}=0 .
\end{array}
$$

The minimization of the left side of Eq. (10) yielded the algebraic equation for the optimal control $\boldsymbol{\Phi}_{a}^{*}$ as:

$$
\frac{\partial L_{C}\left(\hat{\mathbf{X}}, \boldsymbol{\Phi}_{a}^{*}\right)}{\partial \boldsymbol{\Phi}_{a}^{*}}+\mathbf{B}^{T} \frac{\partial V}{\partial \hat{\mathbf{X}}}=0
$$

The optimal control law was obtained by Eq. (11). For example, for the function of quadratic control $L_{C}=g(\hat{\mathbf{X}})+$ $\boldsymbol{\Phi}_{a}^{T} \mathbf{R}_{C} \boldsymbol{\Phi}_{a}$, where $\mathbf{R}_{\mathbf{C}}$ was a positive definite symmetric constant matrix and $g(\hat{\mathbf{X}}) \geq 0$, it was:

$$
\boldsymbol{\Phi}_{a}^{*}=-\frac{1}{2} \mathbf{R}_{C}^{-1} \mathbf{B}^{T} \frac{\partial V}{\partial \hat{\mathbf{X}}} .
$$

By substituting Eq. (12) into Eq. (10), the value function equation was obtained as:

$$
\begin{array}{r}
\frac{\partial V}{\partial t}+\frac{1}{2} \operatorname{tr}\left[\left(\mathbf{R D}^{T} \mathbf{R}_{s}^{-1} \mathbf{D R}+\boldsymbol{\Delta}_{e}^{*} \boldsymbol{\Delta}_{e}^{* T}\right) \frac{\partial^{2} V}{\partial \hat{\mathbf{X}}^{2}}\right] \\
+\left[\mathbf{J} \frac{\partial H_{a}}{\partial \hat{\mathbf{X}}}+\mathbf{C} \hat{\mathbf{X}}+\Delta_{s}^{*}(\hat{\mathbf{X}})+\mathbf{B} \boldsymbol{\Phi}_{a}^{*}\right]^{T} \frac{\partial V}{\partial \hat{\mathbf{X}}} \\
+L_{C}\left(\hat{\mathbf{X}}, \boldsymbol{\Phi}_{a}^{*}\right)=0 .
\end{array}
$$

Equation (13) was solved to obtain $V$, and then the optimal control $\boldsymbol{\Phi}_{a}^{*}$ for the worst-case disturbances was determined by Eq. (12), which was based on the estimated state $\hat{\mathbf{X}}$. The optimally controlled system with uncertain parameters was determined by substituting the control $\boldsymbol{\Phi}_{a}^{*}$ into Eq. (4). The controlled state $\hat{\mathbf{X}}$ was obtained by solving the equation. The statistics of state $\mathbf{X}$ were calculated by using the state $\hat{\mathbf{X}}$ and covariance $\mathbf{R}$, which were used for evaluating control effectiveness. According to the minimax strategy, the stochastic response of the optimally controlled system with non-worst-case disturbances is smaller than that of the optimally controlled system with the worst-case disturbances. Thus, the minimax control for the uncertain nonlinear stochastic system or uncertain quasi-Hamiltonian system with noisy observation was robust.

\section{EXAMPLES AND NUMERICAL RESULTS}

\subsection{Example 1}

To illustrate the application and effectiveness of the proposed stochastic minimax control strategy, first consider the 
control for a main mode vibration of geometrically nonlinear beam with piezoelectric sensor and actuator under random excitation. The cantilever beam under support excitation has the nonlinear stochastic vibration, which can be expressed by the dominant first mode. The modal vibration has the uncertainty of stiffness, damping and excitation, for example, due to other mode effects. The vibration response was estimated by the amplified signal of the sensor with noise. The control was performed by the actuator which can be combined with the beam system. The uncertain nonlinear stochastic system with control and noisy observation was expressed as:

$$
\begin{aligned}
& \ddot{q}+(c+\tilde{c}) \dot{q}+\left(k_{1}+\tilde{k}_{1}\right) q+\left(k_{3}+\tilde{k}_{3}\right) q^{3}= \\
& b \phi_{a}+e_{0} W(t)+\tilde{e}_{w} ; \\
& \phi_{s}=d q+e_{1} W_{s}(t) ;
\end{aligned}
$$

where $q$ was the generalized modal displacement. $\phi_{a}$ was the control. $c, k_{1}$ and $k_{3}$ were respectively the nominal damping, linear stiffness and nonlinear stiffness coefficients. $b$ was the control coefficient. $e_{0}$ was the nominal excitation amplitude. $W$ was the Gaussian white noise with unit intensity. $\phi_{s}$ was the observation. $d$ was the observation coefficient. $e_{1}$ was the observation noise amplitude. $W_{s}$ was the unit Gaussian white noise. $\tilde{c}, \tilde{k}_{1}, \tilde{k}_{3}$ and $\tilde{e}_{w}$ were the uncertain parameters and external disturbance with bounded values $\tilde{c} \in\left[-c^{0}, c^{0}\right]$, $\tilde{k}_{1} \in\left[-k_{1}^{0}, k_{1}^{0}\right], \tilde{k}_{3} \in\left[-k_{3}^{0}, k_{3}^{0}\right]$ and $\tilde{e}_{w} \in\left[-e_{w}^{0}, e_{w}^{0}\right]$. Rewrite Eqs. (14) and (15) as Eqs. (1) and (2), respectively, where $\Phi_{a}=\phi_{a}, \Phi_{s}=\phi_{s}, p=\dot{q}$, and:

$$
\begin{aligned}
& H_{a}=\frac{1}{2} p^{2}+\frac{1}{2} k_{1} q^{2}+\frac{1}{4} k_{3} q^{4} \\
& \mathbf{X}=\left\{\begin{array}{l}
q \\
p
\end{array}\right\} ; \mathbf{C}=\left[\begin{array}{cc}
0 & 0 \\
0 & -c
\end{array}\right] ; \\
& \mathbf{B}=\left\{\begin{array}{l}
0 \\
b
\end{array}\right\} ; \mathbf{F}=\left\{\begin{array}{c}
0 \\
e_{0}
\end{array}\right\} ; \\
& \boldsymbol{\Delta}=\left[\begin{array}{ll}
0 & -\tilde{c} p-\tilde{k}_{1} q-\tilde{k}_{3} q^{3}+\tilde{e}_{w}
\end{array}\right]^{T} ; \\
& \mathbf{D}=\left[\begin{array}{ll}
d & 0
\end{array}\right] ; E=e_{1} \text {. }
\end{aligned}
$$

Applying the extended Kalman filter yielded the differential equation (4) for the estimated state $(\hat{q}, \hat{p})$ with the covariance equation (5). The Hamiltonian system associated with the equation was integrable and non-resonant. To reduce the dimension of the control system and to convert the system state control to system energy control, the stochastic averaging method for integrable Hamiltonian systems was used. Equation (4) was converted into the Itô differential equation for the averaged Hamiltonian ${ }^{29}$ :

$$
\begin{aligned}
d \hat{H}_{a}=\left[m_{h}\left(\hat{H}_{a}\right)+\left\langle\left( b \phi_{a}-\tilde{c} \hat{p}-\tilde{k}_{1} \hat{q}\right.\right.\right. & \left.\left.\left.-\tilde{k}_{3} \hat{q}^{3}+\tilde{e}_{w}\right) \frac{\partial \hat{H}_{a}}{\partial \hat{p}}\right\rangle\right] \mathrm{d} t \\
& +\sigma_{h}\left(\hat{H}_{a}\right) d \xi(t) ; \quad \text { (17) }
\end{aligned}
$$

where $m_{h}$ and $\sigma_{h}$ were respectively the drift and diffusion coefficients. $\xi$ was the unit Wiener process and $\langle\cdot\rangle$ was the averaging operator. According to the minimax stochastic dynamical programming principle, the dynamical programming equation like Eq. (8) could be obtained. The worst-case disturbances (9) and optimal control law (12) became:

$$
\tilde{c}^{*}=-c^{0} \operatorname{sgn}\left(\hat{p}^{2} \frac{d V}{d \hat{H}_{a}}\right) ;
$$

$$
\begin{gathered}
\tilde{k}_{1}^{*}=-k_{1}^{0} \operatorname{sgn}\left(\hat{q} \hat{p} \frac{d V}{d \hat{H}_{a}}\right) ; \\
\tilde{k}_{3}^{*}=-k_{3}^{0} \operatorname{sgn}\left(\hat{q}^{3} \hat{p} \frac{d V}{d \hat{H}_{a}}\right) ; \\
\tilde{e}_{w}^{*}=-e_{w}^{0} \operatorname{sgn}\left(\hat{p} \frac{d V}{d \hat{H}_{a}}\right) . \\
\phi_{a}^{*}=-\frac{b \hat{p}}{2 R_{C}} \frac{d V}{d \hat{H}_{a}} .
\end{gathered}
$$

The corresponding stationary value function equation was:

$$
\begin{aligned}
& \frac{1}{2} \sigma_{h}^{2}\left(\hat{H}_{a}\right) \frac{d^{2} V}{d \hat{H}_{h}^{2}}+m_{h}\left(\hat{H}_{a}\right) \frac{d V}{d \hat{H}_{a}} \\
&+\left\langle c^{0}\left|\hat{p}^{2} \frac{d V}{d \hat{H}_{a}}\right|+k_{1}^{0}\left|\hat{q} \hat{p} \frac{d V}{d \hat{H}_{a}}\right|+k_{3}^{0}\left|\hat{q}^{3} \hat{p} \frac{d V}{d \hat{H}_{a}}\right|\right\rangle \\
&+\left\langle e_{w}^{0}\left|\hat{p} \frac{d V}{d \hat{H}_{a}}\right|\right\rangle-\frac{b^{2}}{4 R_{C}}\left\langle\hat{p}^{2}\right\rangle\left(\frac{d V}{d \hat{H}_{a}}\right)^{2} \\
&+g\left(\hat{H}_{a}\right)=\gamma_{0}
\end{aligned}
$$

where $\gamma_{0}$ was a constant. The optimal control was determined finally by Eqs. (19) and (20). Then the controlled response and its statistics were obtained by solving Eqs. (4) and (15) numerically. The analytical system responses were evaluated by using the probability density, which was obtained by solving the Fokker-Planck-Kolmogorov equation associated with the Itô equation (17). ${ }^{29}$ The efficacy of the proposed optimal control for the uncertain nonlinear stochastic system with noisy observation was evaluated based on the response statistics.

Numerical results of the uncertain control system (14) and observation (15) with non-dimensional parameters $c=1.0$, $k_{1}=10.0, k_{3}=3.0, e_{0}=1.0, b=1.0, d=100.0, e_{1}=0.03$, $c^{0}=0.1 c, k_{1}^{0}=0.1 k_{1}, k_{3}^{0}=0.1 k_{3}, e_{w}{ }^{0}=0.1 R_{11} d / e_{1}$, $R_{C}=1.0$ and the quadratic control coefficient $S_{c 2}=1.0$ (i.e., coefficient of linear term of function $g$ ) were obtained and shown in Figs. 1-7. The control effectiveness of the uncertain system was represented by using the relative response reduction of the proposed stochastic minimax control $K_{m}$, and the control effectiveness by using the stochastic nominal control $K_{n}$ was also given for comparison (the stochastic nominal control was the stochastic optimal control for the corresponding nominal system and the nominal system was the system with nominal parameter values or without considering parametric uncertainty). The probability densities of the controlled and uncontrolled responses of generalized displacement $(q)$ and velocity $(p)$ are shown in Figs. 1(a) and 1(b), respectively. The controlled response probability density near zero is larger than the uncontrolled probability density, and thus the system response is reduced by the proposed control. Figure 2 shows samples of the controlled displacement $(q)$ and velocity $(p)$ responses compared with the uncontrolled responses, which supports the results in Fig. 1. Figure 3 illustrates that the controlled Root-Mean-Square (RMS) displacement response is much smaller than the uncontrolled response, and the controlled RMS response changes slightly with the relative disturbance bound of damping $c^{0} / c$. Figure 4 illustrates that the relative RMS response reduction for the proposed control $K_{m}$ is larger than that for the nominal control $K_{n}$, and the proposed relative response reduction $K_{m}>80 \%$. The stochastic 


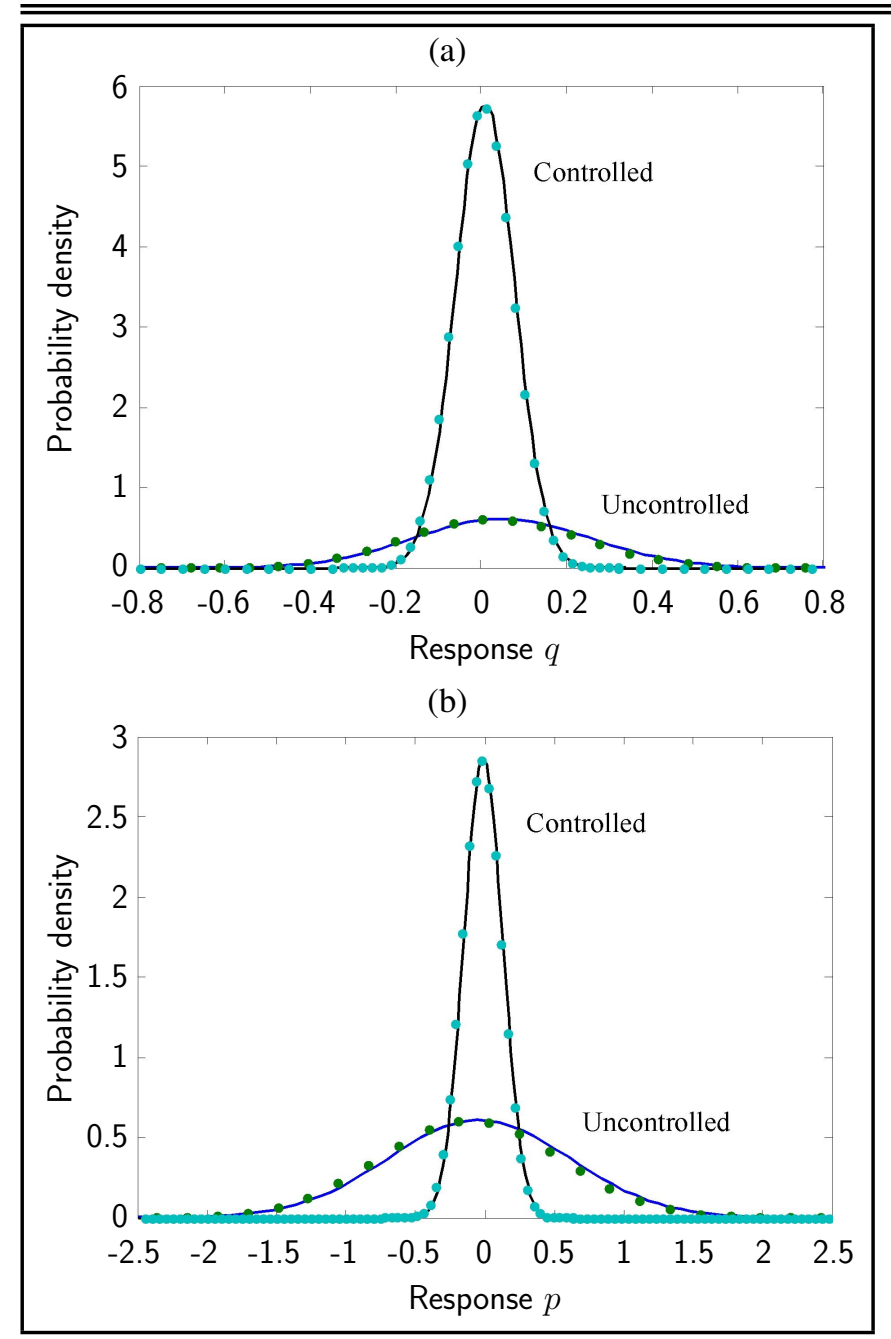

Figure 1. Controlled and uncontrolled probability densities (solid line: analytical; dot: simulated). (a) Probability densities of displacement $q$ and (b) Probability densities of velocity $p$.

response of the uncertain system is reduced largely by using the proposed stochastic minimax control for various relative disturbance bounds of damping $c^{0} / c$.

Figures 5 and 6 illustrate that the relative RMS response reduction using the proposed stochastic minimax control $K_{m}$ is larger than that using the stochastic nominal control $K_{n}$ for the various relative disturbance bounds of linear stiffness $k_{1}^{0} / k_{1}$ and nonlinear stiffness $k_{3}^{0} / k_{3}$, respectively. The proposed relative response reduction $K_{m}$ changes slightly with the relative disturbance bounds. Figure 7 illustrates that the relative RMS response reduction for the proposed control $K_{m}$ is larger than that for the nominal control $K_{n}$ under various relative disturbance bounds of excitation $e_{w}^{0} /\left(R_{11} d / e_{1}\right)$. Therefore, the proposed stochastic minimax control can reduce largely the stochastic vibration response of the uncertain nonlinear system (14) with noisy observation (15), and the control effectiveness has good robustness for various uncertain parameters.

\subsection{Example 2}

To further illustrate the application and effectiveness of the proposed stochastic minimax optimal control strategy, consider the control for a two-main-mode coupling vibration of a geometrically nonlinear beam with piezoelectric sensors and actuators under random excitation. The uncertain nonlinear stochastic system with control and noisy observation was de-

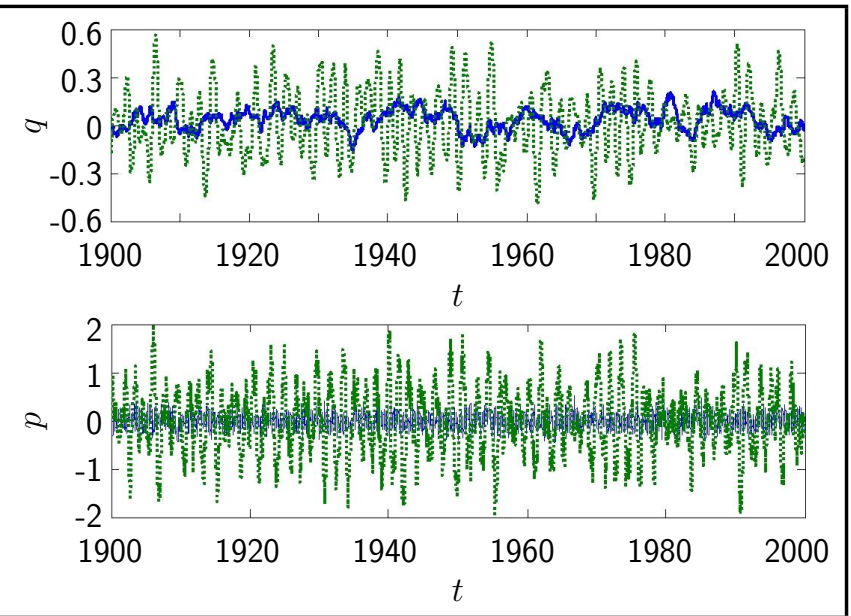

Figure 2. Samples of controlled and uncontrolled responses of displacement $q$ and velocity $p$ (solid line: controlled; dashed line: uncontrolled).

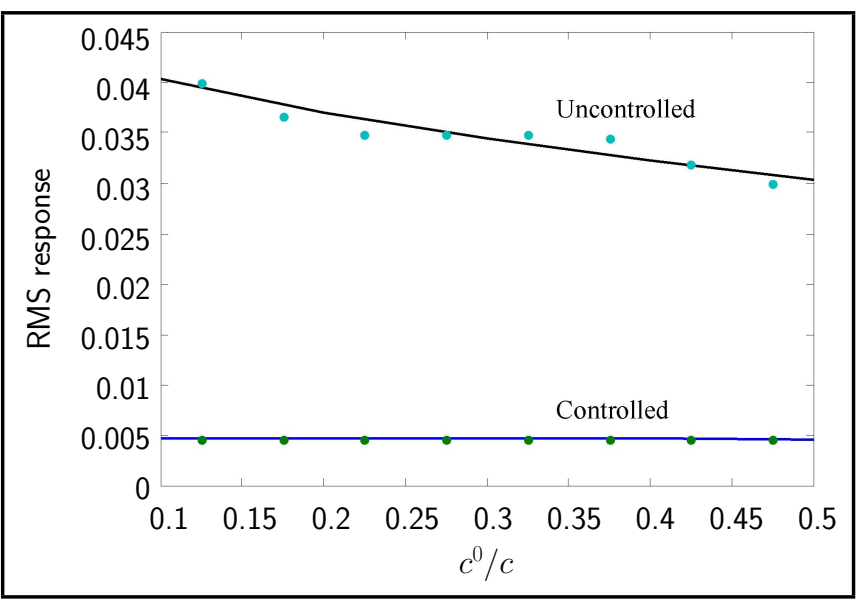

Figure 3. Controlled and uncontrolled RMS responses $q$ for various relative disturbance bounds of damping $c^{0} / c$ (solid line: analytical; dot: simulated).

scribed by Eqs. (1) and (2), in which

$$
\begin{aligned}
& H_{a}=\frac{1}{2}\left(p_{1}^{2}+p_{2}^{2}\right)+\frac{1}{2}\left(k_{11} q_{1}^{2}\right. \\
& \left.+k_{12} q_{2}^{2}\right)+k_{2} q_{1} q_{2}+\frac{1}{4} k_{3}\left(q_{1}-q_{2}\right)^{4} \\
& \mathbf{X}=\left[\begin{array}{llll}
q_{1} & q_{2} & p_{1} & p_{2}
\end{array}\right]^{T} ; \\
& \boldsymbol{\Phi}_{a}=\left[\varphi_{a 1}, \varphi_{a 2}\right]^{T} ; \\
& \boldsymbol{\Phi}_{s}=\left[\varphi_{s 1}, \varphi_{s 2}\right]^{T} \text {; } \\
& \mathbf{C}=\operatorname{diag}\left[0,0,-c_{1},-c_{2}\right] \text {; } \\
& \mathbf{F}=\left[\begin{array}{llll}
0 & 0 & e_{01} & e_{02}
\end{array}\right]^{T} ; \\
& \mathbf{B}=\left[\begin{array}{cccc}
0 & 0 & b_{1} & 0 \\
0 & 0 & 0 & b_{2}
\end{array}\right]^{T} \text {; } \\
& \boldsymbol{\Delta}=\left[\begin{array}{ll}
0 & 0
\end{array}\right. \\
& -\tilde{c}_{1} p_{1}-\tilde{k}_{11} q_{1}-\tilde{k}_{2} q_{2}-\tilde{k}_{3}\left(q_{1}-q_{2}\right)^{3}+\tilde{e}_{w 1} \\
& \left.-\tilde{c}_{2} p_{2}-\tilde{k}_{12} q_{2}-\tilde{k}_{2} q_{1}-\tilde{k}_{3}\left(q_{2}-q_{1}\right)^{3}+\tilde{e}_{w 2}\right]^{T} \text {; } \\
& \mathbf{D}=\left[\begin{array}{llll}
d_{11} & d_{12} & 0 & 0 \\
d_{12} & d_{22} & 0 & 0
\end{array}\right] \\
& \mathbf{E}=\left[\begin{array}{l}
e_{11} \\
e_{12}
\end{array}\right]
\end{aligned}
$$




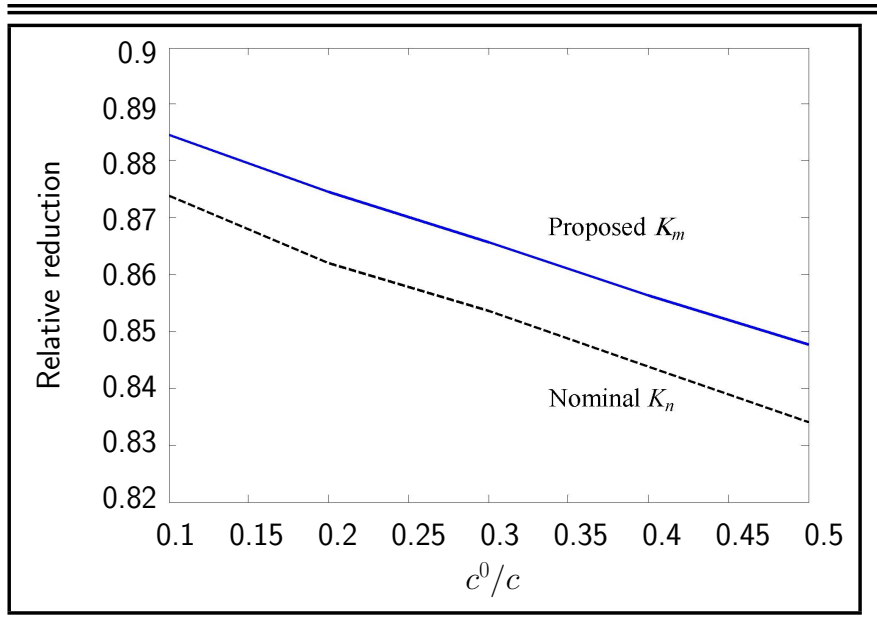

Figure 4. Relative reductions $K_{m}$ and $K_{n}$ of controlled RMS responses $q$ for various relative disturbance bounds of damping $c^{0} / c$.

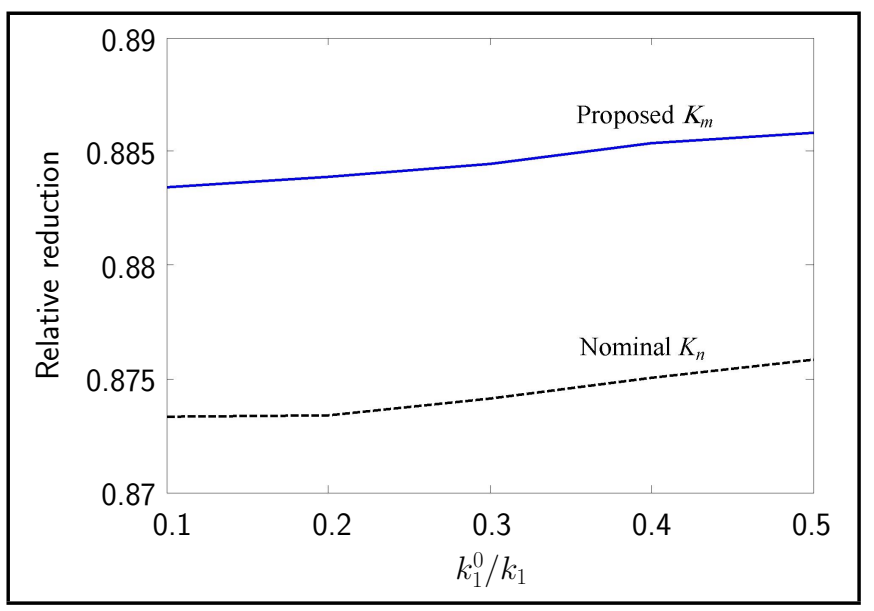

Figure 5. Relative reductions $K_{m}$ and $K_{n}$ of controlled RMS responses $q$ for various relative disturbance bounds of linear stiffness $k_{1}^{0} / k_{1}$.

where $q_{i}$ and $p_{i}(i=1,2)$ were respectively the generalized displacement and momentum, $\phi_{a i}(i=1,2)$ was the control and $\phi_{s i}(i=1,2)$ was the observation. $c_{i}, k_{1 i}, k_{2}$ and $k_{3}(i=$ $1,2)$ were respectively the nominal damping, linear stiffness and nonlinear stiffness coefficients. $b_{i}(i=1,2)$ was the control coefficient. $e_{0 i}(i=1,2)$ was the excitation amplitude. $d_{i j}$ $(i, j=1,2)$ was the observation coefficient and $e_{1 i}(i=1,2)$ was the observation noise amplitude. $\tilde{c}_{i}, \tilde{k}_{1 i}, \tilde{k}_{2}, \tilde{k}_{3}$ and $\tilde{e}_{w i}$ $(i=1,2)$ were the uncertain parameters and external disturbances with bounded values $\tilde{c}_{i} \in\left[-c_{i}^{0}, c_{i}^{0}\right], \tilde{k}_{1 i} \in\left[-k_{1 i}^{0}, k_{1 i}^{0}\right]$, $\tilde{k}_{2} \in\left[-k_{2}^{0}, k_{2}^{0}\right], \tilde{k}_{3} \in\left[-k_{3}^{0}, k_{3}^{0}\right]$ and $\tilde{e}_{w i} \in\left[-e_{w i}^{0}, e_{w i}^{0}\right]$.

According to the proposed procedure, using the extended Kalman filter yielded the differential equation (4) for the estimated state. The Hamiltonian system associated with the equation is non-integrable, and the stochastic averaging method for non-integrable Hamiltonian systems was applied to reduce the dimension of the control system and to convert the system state control to system energy control. Equation (4) was converted into the Itô differential equation, which was similar to Eq. (17), for the averaged Hamiltonian. Using the minimax stochastic dynamical programming principle yielded a dynamical programming equation similar to example 1 . The worst-case disturbances and optimal control law were obtained as:

$$
\tilde{c}_{i}^{*}=-c_{i}^{0} \operatorname{sgn}\left(\hat{p}_{i}^{2} \frac{d V}{d \hat{H}_{a}}\right) ;
$$

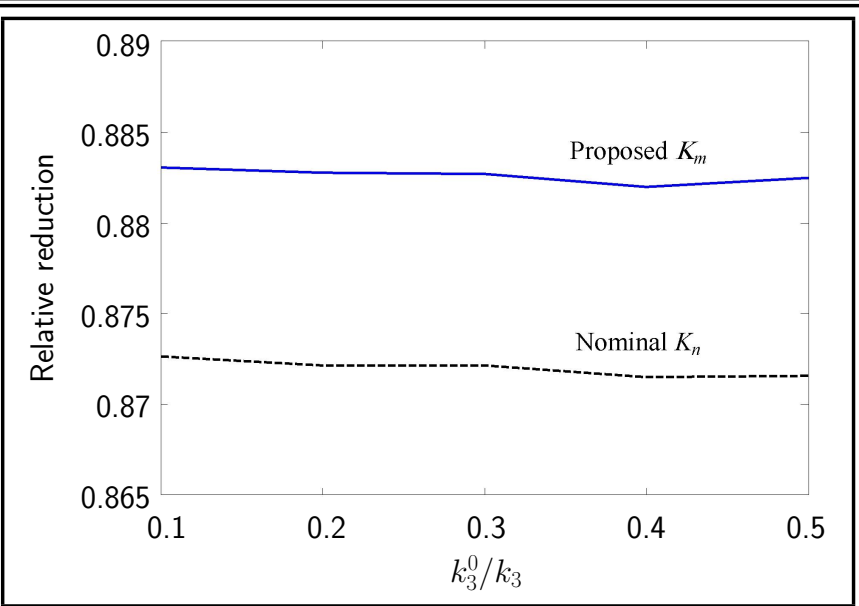

Figure 6. Relative reductions $K_{m}$ and $K_{n}$ of controlled RMS responses $q$ for various relative disturbance bounds of nonlinear stiffness $k_{3}^{0} / k_{3}$.

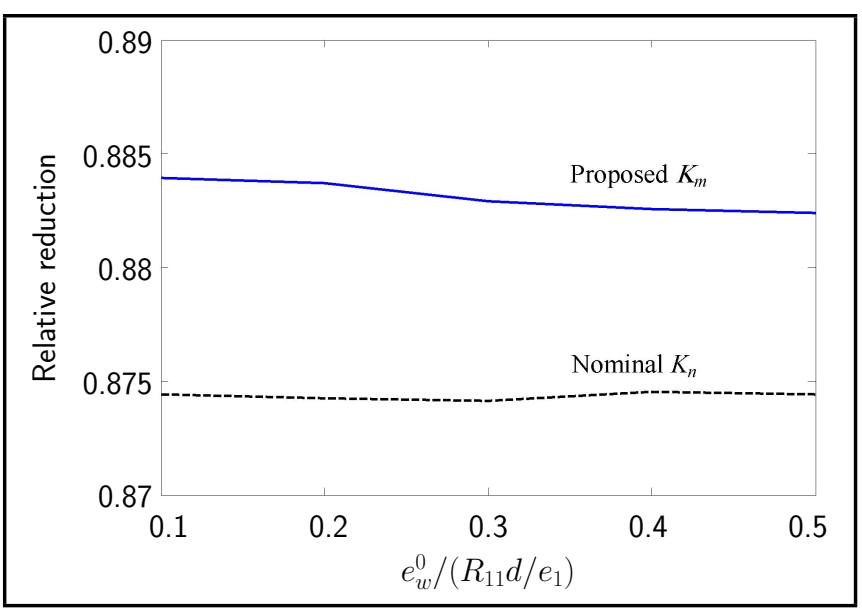

Figure 7. Relative reductions $K_{m}$ and $K_{n}$ of controlled RMS responses $q$ for various relative disturbance bounds of excitation $e_{w}^{0} /\left(R_{11} d / e_{1}\right)$.

$$
\begin{gathered}
\tilde{k}_{1 i}^{*}=-k_{1 i}^{0} \operatorname{sgn}\left(\hat{q}_{i} \hat{p}_{i} \frac{d V}{d \hat{H}_{a}}\right) ; \\
\tilde{k}_{2}^{*}=-k_{2}^{0} \operatorname{sgn}\left(\left(\hat{q}_{1} \hat{p}_{2}+\hat{q}_{2} \hat{p}_{1}\right) \frac{d V}{d \hat{H}_{a}}\right) ; \\
\tilde{k}_{3}^{*}=-k_{3}^{0} \operatorname{sgn}\left(\left(\left(\hat{q}_{1}-\hat{q}_{2}\right)^{3} \hat{p}_{1}+\left(\hat{q}_{2}-\hat{q}_{1}\right)^{3} \hat{p}_{2}\right) \frac{d V}{d \hat{H}_{a}}\right) ; \\
\tilde{e}_{w i}^{*}=-e_{w i}^{0} \operatorname{sgn}\left(\hat{p}_{i} \frac{d V}{d \hat{H}_{a}}\right) ; \\
\phi_{a i}^{*}=-\frac{1}{2} R_{C, i j}^{-1} b_{j} \hat{p}_{j} \frac{d V}{d \hat{H}_{a}} .
\end{gathered}
$$

The corresponding stationary value function equation was:

$$
\begin{aligned}
& \frac{1}{2} \sigma_{h}^{2}\left(\hat{H}_{a}\right) \frac{d^{2} V}{d \hat{H}_{h}^{2}}+m_{h}\left(\hat{H}_{a}\right) \frac{d V}{d \hat{H}_{a}} \\
& +\left\langle c_{i}^{0}\left|\hat{p}_{i}^{2} \frac{d V}{d \hat{H}_{a}}\right|+k_{1 i}^{0}\left|\hat{q}_{i} \hat{p}_{i} \frac{d V}{d \hat{H}_{a}}\right|\right\rangle+ \\
& \quad\left\langle k_{2}^{0}\left|\left(\hat{q}_{1} \hat{p}_{2}+\hat{q}_{2} \hat{p}_{1}\right) \frac{d V}{d \hat{H}_{a}}\right|\right. \\
& \left.+k_{3}^{0}\left|\left(\left(\hat{q}_{1}-\hat{q}_{2}\right)^{3} \hat{p}_{1}+\left(\hat{q}_{2}-\hat{q}_{1}\right)^{3} \hat{p}_{2}\right) \frac{d V}{d \hat{H}_{a}}\right|\right\rangle+
\end{aligned}
$$




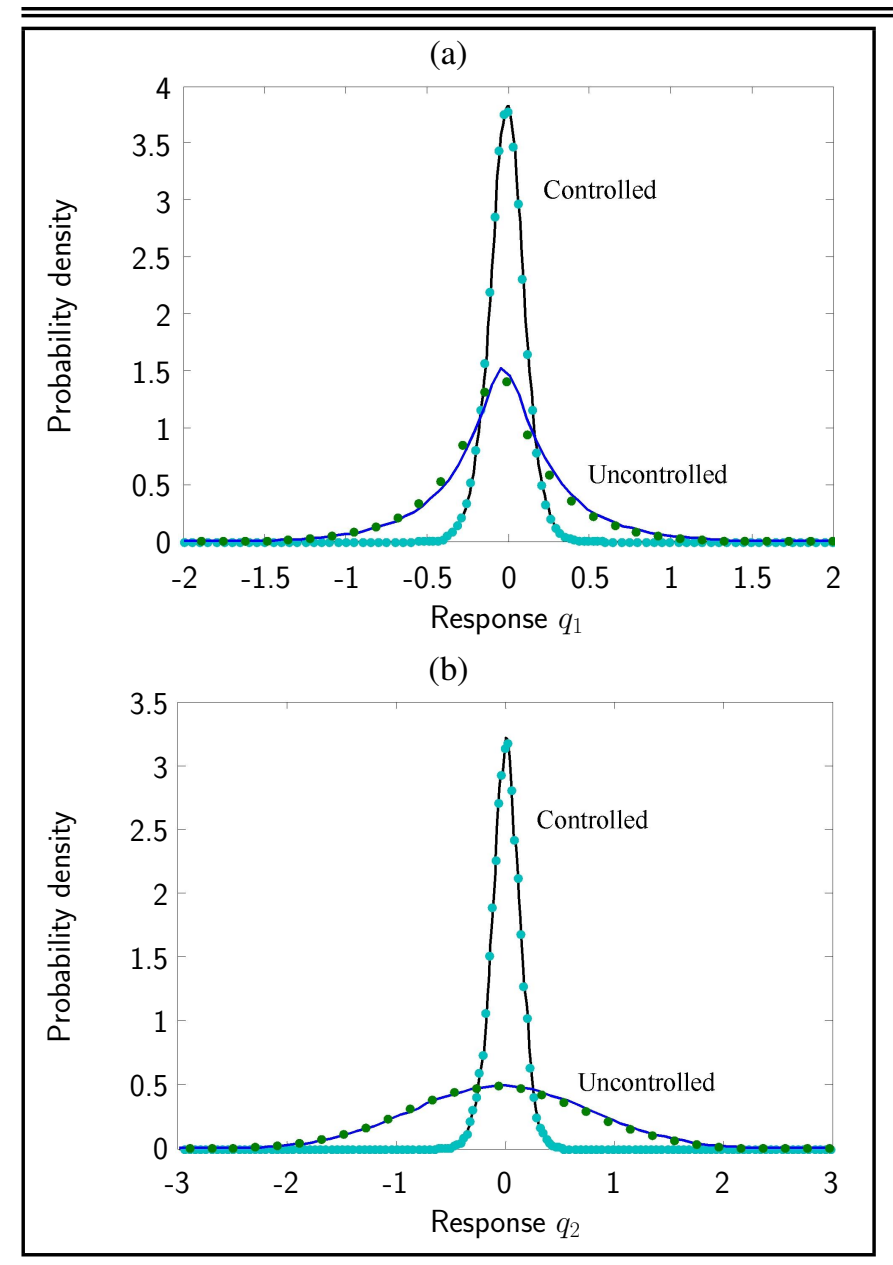

Figure 8. Controlled and uncontrolled probability densities (solid line: analytical; dot: simulated). (a) Probability densities of displacement $q_{1}$ and (b) Probability densities of displacement $q_{2}$.

$$
\begin{array}{r}
\left\langle e_{w i}^{0}\left|\hat{p}_{i} \frac{d V}{d \hat{H}_{a}}\right|\right\rangle-\frac{1}{4}\left\langle b_{i} p_{i} R_{C, i j}^{-1} b_{j} \hat{p}_{j}\right\rangle\left(\frac{\mathrm{d} V}{\mathrm{~d} \hat{H}_{a}}\right)^{2} \\
+g\left(\hat{H}_{a}\right)=\gamma_{0} .
\end{array}
$$

The optimal control was finally determined by Eqs. (23) and (24). Then, the controlled response and its statistics were obtained by either solving Eqs. (4) and (2) or solving the Fokker-Planck-Kolmogorov equation associated with the Itô equation (17). The efficacy of the proposed optimal control for the uncertain nonlinear stochastic system with noisy observation was evaluated based on the response statistics.

Numerical results of the control system and observation with non-dimensional parameters $c_{1}=1.0, c_{2}=5.0, k_{11}=1.0$, $k_{12}=4.0, k_{2}=0.01, k_{3}=1.0, e_{01}=1.0, e_{02}=0.3$, $b_{1}=1.0, b_{2}=0.5, d_{11}=100.0, d_{12}=10.0, d_{22}=80.0$, $e_{11}=0.03, e_{12}=0.03 c_{i}^{0}=0.1 c_{i}, k_{1 i}^{0}=0.1 k_{1 i}, k_{2}^{0}=$ $0.1 k_{2}, k_{3}^{0}=0.1 k_{3}, e_{w i}{ }^{0}=0.0, \mathbf{R}_{C}=\operatorname{diag}[1.0,1.0]$ and the quadratic control coefficient $S_{c 2}=1.0$ (i.e., coefficient of linear term of function $g$ ) were obtained and shown in Figs. 8-13. The control effectiveness for the proposed stochastic minimax control $K_{m}$ and stochastic nominal control $K_{n}$ was defined as in example 1 . The probability densities of the controlled and uncontrolled responses of generalized displacements $q_{1}$ and $q_{2}$ are shown in Figs. 8(a) and 8(b), respectively. The controlled response probability densities near zero are larger than the corresponding uncontrolled probability densities, and thus the system responses are reduced by the proposed con-

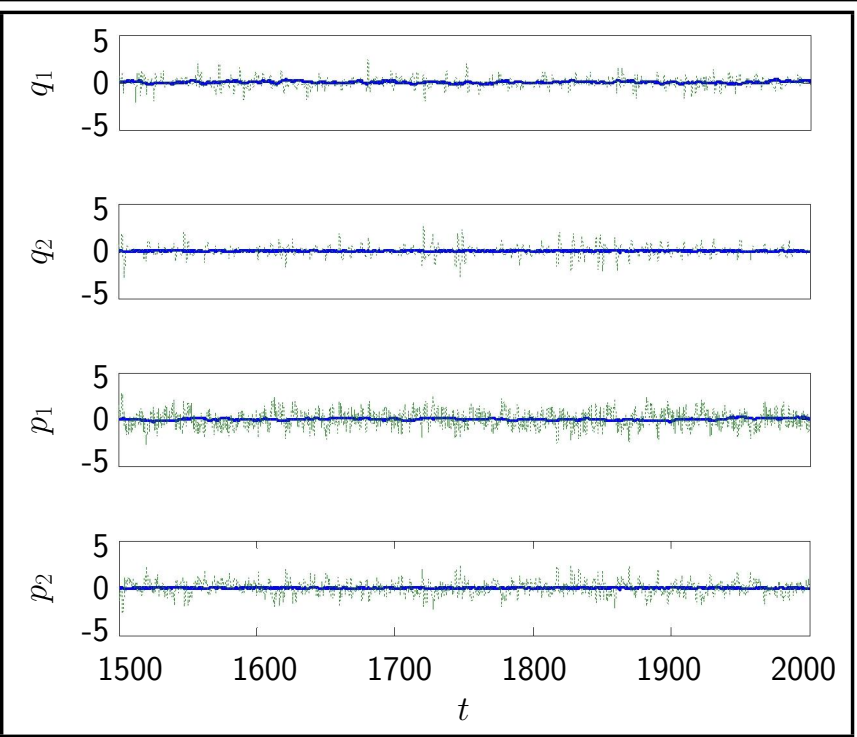

Figure 9. Samples of controlled and uncontrolled responses of displacements $\left(q_{1}, q_{2}\right)$ and velocities $\left(p_{1}, p_{2}\right)$ (solid line: controlled; dashed line: uncontrolled).

trol. Figure 9 shows samples of the controlled displacement $\left(q_{1}\right.$ and $q_{2}$ ) and velocity $\left(p_{1}\right.$ and $p_{2}$ ) responses compared with the uncontrolled responses, in which the response reduction was observed. Figure 10 illustrates that the controlled RMS displacement response $\left(q_{1}\right)$ is much smaller than the uncontrolled response, and the controlled RMS response changes slightly with the relative disturbance bound of damping $c_{1}^{0} / c_{1}$. Figure 11 illustrates that the relative RMS response reduction using the proposed stochastic minimax control $K_{m}$ is larger than that using the stochastic nominal control $K_{n}$ for various relative disturbance bounds of damping $c_{1}^{0} / c_{1}$. Figures 12 and 13 illustrate that the relative RMS response reduction, using the proposed control $K_{m}$, is larger than that using the nominal control $K_{n}$ for various relative disturbance bounds of linear stiffness $k_{11}^{0} / k_{11}$ and nonlinear stiffness $k_{3}^{0} / k_{3}$, respectively. The proposed relative response reduction $K_{m}$ varying with the relative disturbance bound of nonlinear stiffness is larger than the others. It is obtained again that the proposed stochastic minimax control can reduce remarkably the stochastic vibration response of the uncertain nonlinear system with noisy observation (21), and the control effectiveness is robust for uncertain parameters except nonlinear stiffness.

\section{CONCLUSIONS}

The stochastic minimax control strategy for the uncertain nonlinear quasi-Hamiltonian systems with noisy observations under random excitations has been proposed based on the extended Kalman filter and minimax stochastic dynamical programming principle. The control strategy includes three parts as follows: the uncertain nonlinear stochastic quasiHamiltonian system with noisy observation is first converted into the completely observed nonlinear stochastic system with the uncertain parameters for the estimated state based on the extended Kalman filter. The dual dynamical programming equation is derived based on the minimax stochastic dynamical programming principle. The worst-case disturbances are determined for bounded uncertain parameters. Lastly, the optimal control law is determined for the worst case and estimated state by the programming equation. The proposed minimax control 


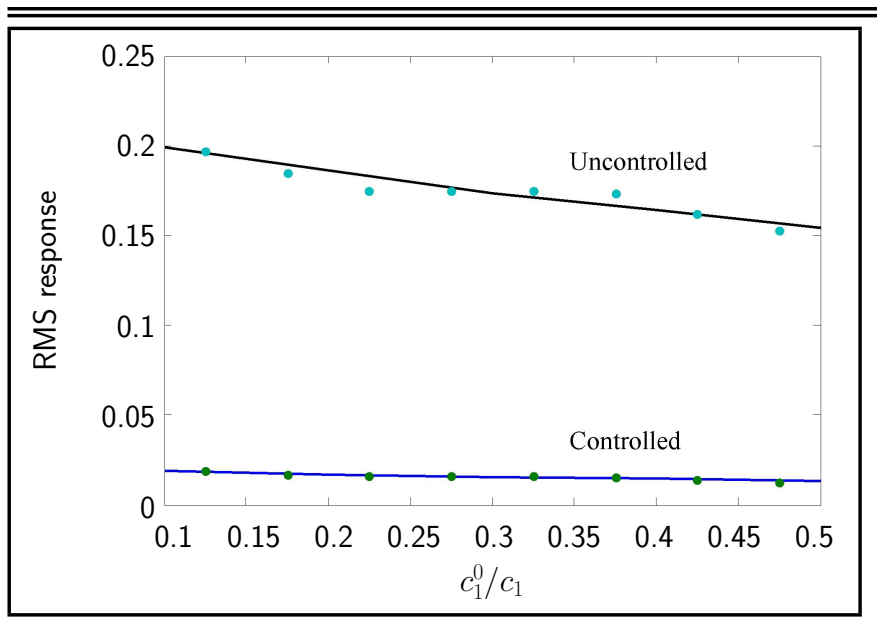

Figure 10. Controlled and uncontrolled RMS responses $q_{1}$ for various relative disturbance bounds of damping $c_{1}^{0} / c_{1}$ (solid line: analytical; dot: simulated).

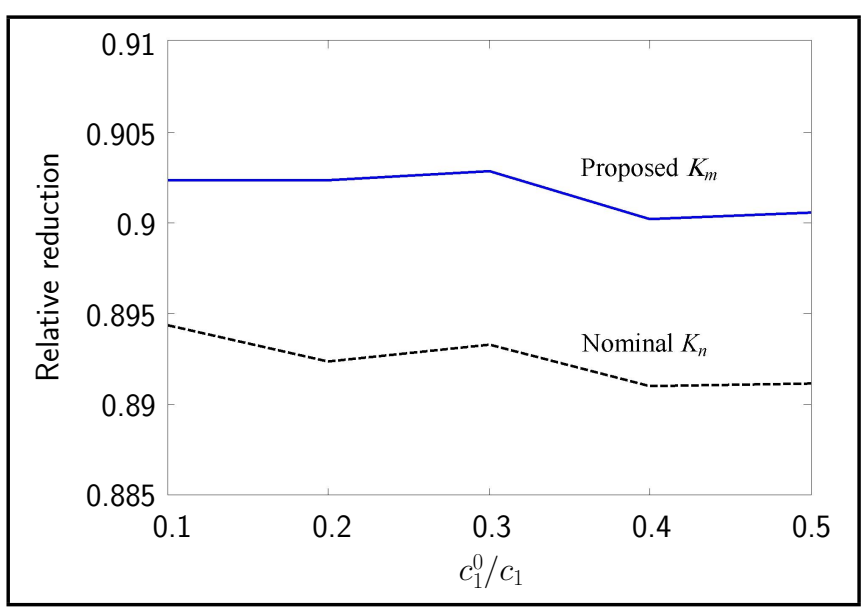

Figure 11. Relative reductions $K_{m}$ and $K_{n}$ of controlled RMS responses $q_{1}$ for various relative disturbance bounds of damping $c_{1}^{0} / c_{1}$.

strategy is applicable to various uncertain nonlinear stochastic multi-degree-of-freedom systems with noisy observations.

The proposed minimax control strategy has been applied to the vibration control of two uncertain nonlinear stochastic systems with noisy observations. Numerical results have illustrated that the proposed minimax control can reduce largely the stochastic vibration response of uncertain nonlinear systems with noisy observations, and the control effectiveness is insensitive to varying system parameters such as observation and nonlinear coefficients. Thus, the proposed stochastic minimax control strategy is feasible and effective for the vibration response reduction of uncertain nonlinear structure systems with noisy observations under random excitations.

\section{ACKNOWLEDGEMENTS}

This work was supported by the National Natural Science Foundation of China under grant nos. 11572279, 11432012, 11772293 and 11621062.

\section{REFERENCES}

1 Housner, G. W., Bergman, L. A., Caughey, T. K., Chassiakos, A. G., Claus, R. O., Masri, S. F., Skelton, R. E., Soong, T. T., Spencer, B. F. and Yao, J. T. P. Structural control: past, present, and fu-

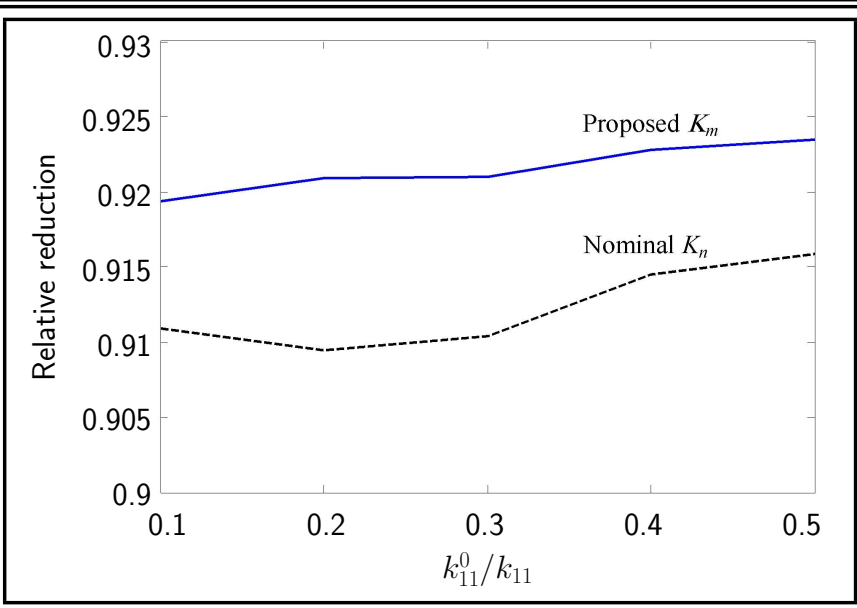

Figure 12. Relative reductions $K_{m}$ and $K_{n}$ of controlled RMS responses $q_{1}$ for various relative disturbance bounds of linear stiffness $k_{11}^{0} / k_{11}$.

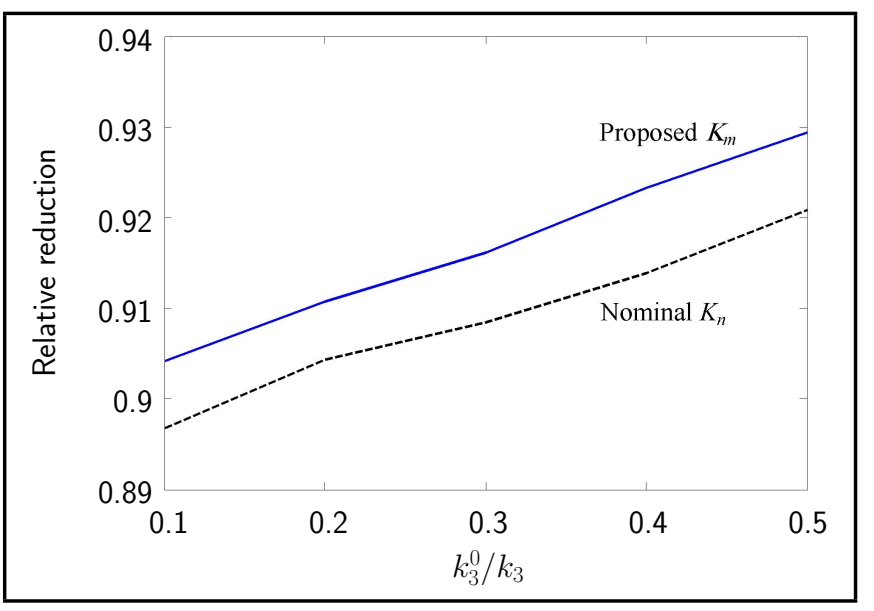

Figure 13. Relative reductions $K_{m}$ and $K_{n}$ of controlled RMS responses $q_{1}$ for various relative disturbance bounds of nonlinear stiffness $k_{3}^{0} / k_{3}$.

ture, ASCE Journal of Engineering Mechanics, 123, 897-971, (1997). https://dx.doi.org/10.1061/(ASCE)07339399(1997)123:9(897)

2 Soong, T. T. and Spencer, B. F. Supplemental energy dissipation: state-of-the-art and state-of-thepractice, Engineering Structures, 24, 243-259, (2002). https://dx.doi.org/10.1016/S0141-0296(01)00092-X

3 Soong, T. T. and Cimellaro, G. P. Future directions in structural control, Structural Control and Health Monitoring, 16, 7-16, (2009). https://dx.doi.org/10.1002/stc.291

4 Casciati, F., Rodellar, J. and Yildirim, U. Active and semi-active control of structures -theory and application: a review of recent advances, Journal of Intelligent Material Systems and Structures, 23, 1181-1195, (2012). https://dx.doi.org/10.1177/1045389X12445029

5 Spencer, B. F. and Nagarajaiah, S. State of the art of structural control, ASCE Journal of Structural Engineering, 129, 845-856, (2003). https://dx.doi.org/10.1061/(ASCE)07339445(2003)129:7(845)

6 Dyke, S. J., Spencer, B. F., Sain, M. K. and Carlson, J. D. Modeling and control of magnetorheological dampers for seismic response reduction, Smart Materials and Structures, 5, 565-575, (1996). 
7 York, D., Wang, X. and Gordaninejad, F. A new MR fluidelastomer vibration isolator, Journal of Intelligent Material Systems and Structures, 18, 1221-1225, (2007).

8 Zhou, G. Y. and Wang, Q. Study on the adjustable rigidity of magnetorheological-elastomer-based sandwich beams, Smart Materials and Structures, 15, 59-74, (2006). https://dx.doi.org/10.1177/1045389X07083622

9 Choi, W. J., Xiong, Y. P. and Shenoi, R. A. Vibration characteristics of sandwich beam with steel skins and magnetorheological elastomer cores, Advances in Structural Engineering, 13, 837-847, (2010). https://dx.doi.org/10.1260/1369-4332.13.5.837

10 Rajamohan, V., Rakheja, S. and Sedaghati, R. Vibration analysis of a partially treated multi-layer beam with magnetorheological fluid, Journal of Sound and Vibration, 329, 3451-3469, (2010). https://dx.doi.org/10.1016/j.jsv.2010.03.010

11 Ying, Z. G., Ni, Y. Q. and Huan, R. H. Stochastic microvibration response analysis of a magnetorheological viscoelastomer based sandwich beam under localized magnetic fields, Applied Mathematical Modelling, 39, 5559-5566, (2015). https://dx.doi.org/10.1016/j.apm.2015.01.028

12 Nayak, B., Dwivedy, S. K. and Murthy, K. S. R. K. Dynamic analysis of magnetorheological elastomer-based sandwich beam with conductive skins under various boundary conditions, Journal of Sound and Vibration, 330, 18371859, (2011). https://dx.doi.org/10.1016/j.jsv.2010.10.041

13 Ying, Z. G., Ni, Y. Q. and Duan, Y. F. Stochastic vibration suppression analysis of an optimal bounded controlled sandwich beam with MR visco-elastomer core, Smart Structures and Systems, 19, 21-31, (2017). https://dx.doi.org/10.12989/sss.2017.19.1.021

14 Rao, S. S. and Sunar, M. Piezoelectricity and its use in disturbance sensing and control of flexible structures: a survey, ASME Applied Mechanics Reviews, 47, 113-123, (1994). https://dx.doi.org/10.1115/1.3111074

15 Narayanan, S. and Balamurugan,V. Finite element modelling of piezolaminated smart structures for active vibration control with distributed sensors and actuators, Journal of Sound and Vibration, 262, 529-562, (2003). https://dx.doi.org/10.1016/S0022-460X(03)00110-X

16 Ray, M. C. Optimal control of laminated shells using piezoelectric sensor and actuator layers, AIAA Journal, 41, 1151-1157, (2003). https://dx.doi.org/10.2514/2.2058

17 Gupta, V. K., Seshu, P. and Issac, K. K. Finite element and experimental investigation of piezoelectric actuated smart shells, AIAA Journal, 42, 2112-2123, (2004). https://dx.doi.org/10.2514/1.2902

18 To, C. W. S. and Chen, T. Optimal control of random vibration in plate and shell structures with distributed piezoelectric components, International Journal of Mechanical Sciences, 49, 1389-1398, (2007). https://dx.doi.org/10.1016/j.ijmecsci.2007.03.015
19 Jin, Z. L., Yang, Y. W. and Soh, C. K. Semianalytical solutions for optimal distributions of sensors and actuators in smart structure vibration control, Smart Structures and Systems, 6, 767-792, (2010). https://dx.doi.org/10.12989/sss.2010.6.7.767

20 Ying, Z. G., Feng, J., Zhu, W. Q. and Ni, Y. Q. Stochastic optimal control analysis of a piezoelectric shell subjected to stochastic boundary perturbations, Smart Structures and Systems, 9, 231-251, (2012). https://dx.doi.org/10.12989/sss.2012.9.3.231

21 Vepa, R. Dynamics of Smart Structures, John Wiley \& Sons, Chichester, (2010).

22 Fisco, N. R. and Adeli, H. Smart structures: part I -active and semi-active control, Scientia Iranica A, 18, 275-284, (2011). https://dx.doi.org/10.1016/j.scient.2011.05.034

23 Fisco, N. R. and Adeli, H. Smart structures: part II -hybrid control systems and control, Scientia Iranica A, 18, 285-295, (2011). https://dx.doi.org/10.1016/j.scient.2011.05.035

24 Stengel, R. F. Optimal Control and Estimation, John Wiley \& Sons, New York, (1994).

25 Yong, J. M. and Zhou, X. Y. Stochastic Controls, Hamiltonian Systems and HJB Equations, Springer-Verlag, New York, (1999).

26 Fleming, W. H. and Soner, H. M. Controlled Markov Processes and Viscosity Solutions, Springer, New York, (2006).

27 Bensoussan, A. Stochastic Control of Partially Observable Systems, Cambridge University Press, Cambridge, (1992).

28 Anderson, B. D. O. and Moore, J. R. Optimal Filtering, Dover Publication, New York, (2005).

29 Zhu, W. Q. Nonlinear stochastic dynamics and control in Hamiltonian formulation, ASME Applied Mechanics Reviews, 59, 230-248, (2006). https://dx.doi.org/10.1115/1.2193137

30 Socha, L. Linearization in analysis of nonlinear stochastic systems: recent results -part II: application, ASME Applied Mechanics Reviews, 58, 303-315, (2005). https://dx.doi.org/10.1115/1.1896368

31 Datta, T. K. A brief review of stochastic control of structures, Proc. International Symposium on Engineering under Uncertainty, Springer, India, 119-139, (2013). https://dx.doi.org/10.1007/978-81-322-0757-3_6

32 Agrawal, A. K. and Yang, J. N. Optimal polynomial control of seismic-excited linear structures, ASCE Journal of Engineering Mechanics, 122, 753761, (1996). https://dx.doi.org/10.1061/(ASCE)07339399(1996)122:8(753)

33 Nagarajaiah, S. Adaptive passive, semiactive, smart tuned mass dampers: identification and control using empirical mode decomposition, Hilbert transform, and short-term Fourier transform, Structural Control and Health Monitoring, 16, 800-841, (2009). https://dx.doi.org/10.1002/stc.349 
${ }^{34} \mathrm{Li}$, J., Peng, Y. B. and Chen, J. B. A physical approach to structural stochastic optimal controls, Probabilistic Engineering Mechanics, 25, 127-141, (2010). https://dx.doi.org/10.1016/j.probengmech.2009.08.006

35 Aldemir, U. Predictive suboptimal semiactive control of earthquake response, Structural Control and Health Monitoring, 17, 654-674, (2010). https://dx.doi.org/10.1002/stc.342

36 Ying, Z. G. and Ni, Y. Q. Optimal control for vibration peak reduction via minimizing large responses, Structural Control and Health Monitoring, 22, 826-846, (2015). https://dx.doi.org/10.1002/stc.1722

37 Socha, L. A. Application of true linearization in stochastic quasi-optimal control problems, Structural Control and Health Monitoring, 7, 219-230, (2000). https://dx.doi.org/10.1002/stc.4300070207

38 Dimentberg, M. F., Iourtchenko, A. S. and Brautus, A. S. Optimal bounded control of steady-state random vibrations, Probabilistic Engineering Mechanics, 15, 381-386, (2000). https://dx.doi.org/10.1016/S0266-8920(00)00008-4

39 Dimentberg, M. F. and Bratus, A. S. Bounded parametric control of random vibrations, Proceedings of Royal Society London A, 456, 2351-2363, (2000). https://dx.doi.org/10.1098/rspa.2000.0615

${ }^{40}$ Ying, Z. G., Ni, Y. Q. and Duan, Y. F. Parametric optimal bounded feedback control for smart parameter-controllable composite structures, Journal of Sound and Vibration, 339, 38-55, (2015). https://dx.doi.org/10.1016/j.jsv.2014.11.018

${ }^{41}$ Crespo, L. G. and Sun, J. Q. Stochastic optimal control of nonlinear dynamical systems via Bellman's principle and cell mapping, Automatica, 39, 2109-2114, (2003). https://dx.doi.org/10.1023/A:1015600430713

42 Park, J. H. and Min, K. W. Bounded nonlinear stochastic control based the probability distribution for sdof oscillator, Journal of Sound and Vibration, 281, 141-153, (2005). https://dx.doi.org/10.1016/j.jsv.2004.01.008

${ }^{43}$ Scruggs, J. T., Taflanidis, A. A. and Iwan, W. D. Nonlinear stochastic controllers for semiactive and regenerative systems with guaranteed quadratic performance bounds part 1: state feedback control, Structural Control and Health Monitoring, 14, 1101-1120, (2007). https://dx.doi.org/10.1002/stc.196

${ }^{44}$ Ying, Z. G. and Zhu, W. Q. A stochastic optimal control strategy for partially observable nonlinear quasi-Hamiltonian systems, Journal of Sound and Vibration, 310, 184-196, (2008). https://dx.doi.org/10.1016/j.jsv.2007.07.065

45 Ying, Z. G., Hu, R. C. and Huan, R. H. Optimal vibration control for structural quasi-Hamiltonian systems with noised observations, International Journal of Acoustics and Vibration, 22, 233-241, (2017). https://dx.doi.org/10.20855/ijav.2017.22.2469

46 Ying, Z. G. and Zhu, W. Q. Optimal bounded control for nonlinear stochastic smart structure systems based on extended Kalman filter, Nonlinear Dynamics, 90: 105-114, (2017). https://dx.doi.org/10.1007/s11071-017-3650-7
47 Xu, Y., Liu, Q., Guo, G., Xu, C. and Liu, D. Dynamical responses of airfoil models with harmonic excitation under uncertain disturbance, Nonlinear Dynamics, 89: 1579-1590, (2017). https://dx.doi.org/10.1007/s11071017-3536-8

48 Wang, Z. Q., Xu, Y. and Yang, H. Levy noise induced stochastic resonance in an FHN model, Science China, Technological Sciences, 59: 371-375, (2016). https://dx.doi.org/10.1007/s11431-015-6001-2

49 Zhou, K. M., Doyle, J. C. and Glover, K. Robust and Optimal Control, Prentice-Hall, New Jersey, (1996).

50 Basar, T. and Bernhard, P. $H^{\text {inf }}$-Optimal Control and Related Minimax Design Problems: A Dynamic Game Approach, Birkhauser, Boston, (2008). https://dx.doi.org/1007/978-0-817-4757-5

51 Boltyanski, V. G. and Poznyak, A. S. The Robust Maximum Principle: Theory and Application, Springer, New York, (2012).

52 Stengel, R. F. and Ryan, L. E. Stochastic robustness of linear time-invariant control systems, IEEE Transactions on Automatic Control, 36, 82-87, (1991). https://dx.doi.org/10.1109/9.62270

${ }^{53}$ Looze, D., Poor, H., Vastola, K. and Darragh, J. Minimax control of linear stochastic systems with noise uncertain, IEEE Transactions on Automatic Control, 28, 882888, (1983). https://dx.doi.org/10.1109/TAC.1983.1103353

54 Savkin, A. V. and Petersen, I. R. Minimax optimal control of uncertain systems with structured uncertainty, International Journal of Robust and Nonlinear Control, 5, 119137, (1995). https://dx.doi.org/10.1002/rnc.4590050204

55 Ugrinovskii, V. A. Robust $\mathrm{H}^{\text {inf }}$ control in the presence of stochastic uncertainty, International Journal of Control, 71, 219-237, (1998). https://dx.doi.org/10.1080/002071798221849

56 Poznyak, A. S., Duncan, T. E., Duncan, B. P. and Boltyansky, V. G. Robust optimal control for minimax stochastic linear quadratic problem, International Journal of Control, 75, 1054-1065, (2002). https://dx.doi.org/10.1080/00207170210156242

57 Ying, Z. G. A minimax stochastic optimal control for bounded-uncertain systems, Journal of Vibration and Control, 16, 1591-1604, (2010). https://dx.doi.org/10.1177/1077546309103282

$58 \mathrm{Hu}$, R. C., Ying, Z. G. and Zhu, W. Q. Stochastic minimax optimal control strategy for uncertain quasi-Hamiltonian systems using stochastic maximum principle, Structural and Multidisciplinary Optimization, 49, 69-80, (2014). https://dx.doi.org/10.1007/s00158-013-0958-x

59 Mase, G. E. Continuum Mechanics, McGraw-Hill, New York, (1970).

60 Ying, Z. G. and Ni, Y. Q. Dynamic asymmetry of piezoelectric shell structures, Journal of Sound and Vibration, 332, 3706-3723, (2013). https://dx.doi.org/10.1016/j.jsv.2013.03.002 